\title{
Kinetics of the B-A transition of DNA: analysis of potential contributions to a reaction barrier
}

\author{
Dietmar Porschke ${ }^{1}$
}

Received: 21 July 2017 / Revised: 21 November 2017 / Accepted: 2 January 2018 / Published online: 5 February 2018

(c) The Author(s) 2018. This article is an open access publication

\begin{abstract}
Because of open problems in the relation between results obtained by relaxation experiments and molecular dynamics simulations on the B-A transition of DNA, relaxation measurements of the B-A dynamics have been extended to a wider range of conditions. Field-induced reaction effects are measured selectively by the magic angle technique using a novel cell construction preventing perturbations from cell window anisotropy. The kinetics was recorded for the case of poly[d(AT)] up to the salt concentration limit of $4.4 \mathrm{mM}$, where aggregation does not yet interfere. Now experimental data on the B-A dynamics are available for poly[d(AT)] at salt concentrations of $0.18,0.73,2.44$ and $4.4 \mathrm{mM}$. In all cases, a spectrum of time constants is found, ranging from $\sim 10 \mu \mathrm{s}$ up to components approaching $\sim 1 \mathrm{~ms}$. The relatively small dependence of these data on the salt concentration indicates that electrostatic effects on the kinetics are not as strong as may be expected. The ethanol content at the transition center is a linear function of the logarithm of the salt concentration, and the slope is close to that expected from polyelectrolyte theory. The B-A transition dynamics was also measured in $\mathrm{D}_{2} \mathrm{O}$ at a salt concentration of $2.4 \mathrm{mM}$ : the center of the transition is found at $20.0 \mathrm{~mol} / 1 \mathrm{H}_{2} \mathrm{O}$ and at $20.1 \mathrm{~mol} / 1 \mathrm{D}_{2} \mathrm{O}$ with an estimated accuracy of $\pm 0.1 \mathrm{~mol} / \mathrm{l}$; the spectrum of time constants at the respective transition centers is very similar. The experimental results are discussed regarding the data obtained by molecular dynamics simulations.
\end{abstract}

Keywords B-A transition · Kinetics · Magic angle measurements $\cdot$ Electrostatics $\cdot$ Solvent isotope effect

\section{Introduction}

Looking at molecular models of the B- and A-form DNA suggests that the transition between these well-ordered, right-handed double-helical structures (Saenger 1984) is a relatively simple rearrangement. The molecular details of Band A-helix structures in crystals have been analyzed at high resolution (Egli et al. 1998; Schneider et al. 1998; Dickerson and $\mathrm{Ng}$ 2001; Ng and Dickerson 2002). A short summary of the major differences: (1) the helix increase per base pair is $3.4 \AA$ in the $\mathrm{B}$ form and $\sim 2.9 \AA$ in the A form; (2) the planes of the base pairs are inclined with respect to the helix axis at angles of $\sim 90^{\circ}$ for the $\mathrm{B}$ and of $74^{\circ}$ for the $\mathrm{A}$ form; (3) the

Special Issue: Chemical Kinetics, Biological Mechanisms and Molecular Evolution.

Dietmar Porschke

dpoersc@gwdg.de

1 Max Planck Institute for Biophysical Chemistry, 37077 Göttingen, Germany sugar pucker is $\mathrm{C}^{\prime}$-endo for the $\mathrm{B}$ and $\mathrm{C} 33^{\prime}$-endo for the $\mathrm{A}$ form; (4) the hydration is more extensive in the B form and more "economic" in the A form (Saenger et al. 1986). Dickerson and $\mathrm{Ng}(2001,2002)$ concluded from their analysis of the structures that there is a smooth transition between the $\mathrm{B}$ and $\mathrm{A}$ forms without major activation barriers.

The equilibrium conditions for the existence of the Band A-form DNA in solution have been characterized in detail (Ivanov et al. 1974). In general, the B form is observed in usual aqueous solutions, whereas formation of the A form requires reduction of the water activity, e.g., by addition of ethanol. Because addition of ethanol not only favors the A form but also aggregation and precipitation of DNA, such reactions had to be avoided by reduction of the salt concentration. These are the general boundary conditions for experimental studies of the B-A transition in solution-obviously valid for studies of both the equilibrium and kinetics. Boundary conditions and the high rate of the B-A transition restrict the experimental potential for analysis of the kinetics. The dynamics of the B-A transition could only be analyzed by the electric field jump technique (Jose and Porschke 
2004, 2005). Electric field pulses induce a reaction from the A toward the B form. Experimental evidence (Jose and Porschke 2004) indicates that the A $\rightarrow$ B reaction is driven by "dipolar stretching;" a dipole increase is expected upon the $\mathrm{A} \rightarrow \mathrm{B}$ reaction resulting from an increase of the contour length. A contribution to the driving force may also come from a dissociation field effect (Onsager 1934). The B $\rightarrow$ A reaction recorded at zero field strength (after electric field pulses) always showed a spectrum of time constants with a major amplitude at $\sim 10 \mu$ s followed by slower components with time constants not larger than $\sim 1 \mathrm{~ms}$.

The dynamics of the B-A transition has been studied more extensively by molecular dynamics simulations than by experiments in solution. Initial simulations suggested that the transition proceeds within $\sim 1 \mathrm{~ns}$ in both directions. Further simulations with accepted force fields indicated that the transition is observed only in the direction from the A to the $B$ form in aqueous solutions with time constants of $\sim 1 \mathrm{~ns}$, whereas the opposite reaction from the B to the A form, expected to occur at reduced water activity, has not been observed under these conditions yet (Cheatham and Kollman 1996; Cheatham et al. 1997; Sprous et al. 1998; Noy et al. 2007; Knee et al. 2008). Meanwhile the conclusion about a low rate for the B-A transition at, for example, 85 volume\% ethanol (vol\% EtOH) appears to be accepted in general. Apparently, there is a reaction barrier for the B-A transition at reduced water activity. Cheatham et al. (1997) observed a reaction from the B to A form in $85 \mathrm{vol} \% \mathrm{EtOH}$ when the $\mathrm{C}^{\prime}$-endo sugar pucker was stabilized, and Knee et al. (2008) observed the reaction from the B to the A form, when "waters were restrained in the major groove of B DNA." In summary, the simulations indicate the existence of a reaction barrier under the conditions of reduced water activity.

The present investigation was executed with the goal to obtain more detailed information about contributions to the activation barrier. The experimental data obtained in the preceding investigations were restricted to a limited range of low salt concentrations, which may be a serious problem in particular for a reaction of a polyelectrolyte. Thus, an extension of the data on the B-A dynamics to higher salt concentrations should be useful. Poly[d(AT)] was selected for these tests because its B-A transition appears in a very narrow range of solvent conditions and thus can be characterized at a higher accuracy than for natural DNAs with mixed sequences. Another favorable property of poly[d(AT)] for this analysis is a relatively high solubility at reduced water activity.

Because the B-A transition is induced by reduction of the water activity and hydration of the double helix is essential for the transition, effects upon replacement of $\mathrm{H}_{2} \mathrm{O}$ by $\mathrm{D}_{2} \mathrm{O}$ may provide information about the nature of interactions. Such analysis is also suggested by the possibility "that the barrier for the transition lies in the organization of the solvent" (Knee et al. 2008).

\section{Materials and methods}

Poly[d(A-T)] from Sigma was dialyzed extensively, first against a high salt buffer with $0.2 \mathrm{M} \mathrm{NaCl}, 1 \mathrm{mM}$ cacodylate $\mathrm{pH} 7.0,1 \mathrm{mM}$ EDTA and finally against $250 \mu \mathrm{M}$ $\mathrm{NaCl}, 250 \mu \mathrm{M}$ cacodylate $\mathrm{pH}$ 7.0, $50 \mu \mathrm{M}$ EDTA. Solutions were prepared for measurements by mixing DNA, salt and buffer first; ethanol was added in the last step, except for minor water quantities for filling up to the desired volume. Quantities were controlled by pipetting and weighing. Samples exposed to electric field pulses must be bubble free to avoid cavitation. Thus, these samples had to be degassed under vacuum after filling them into the field jump cells. Because some reduction of the ethanol content cannot be avoided during this procedure, the ethanol content was determined after completion of the field jumps by measurement of the density in a densitometer DMA 602 (Anton Paar, Graz, Austria). UV spectra of the solutions in the cell were recorded directly after filling the cell and after completion of the measurements in a Perkin-Elmer Lambda 17. Conductivities were also determined. The buffers always contained $1 \mathrm{mM}$ Na-cacodylate $\mathrm{pH} 7$ and $0.2 \mathrm{mM}$ EDTA; in addition, there was $1 \mathrm{mM} \mathrm{NaCl}$ in buffer A, $3 \mathrm{mM} \mathrm{NaCl}$ in buffer $\mathrm{B}$ and $10 \mathrm{mM} \mathrm{NaCl}$ in buffer C. All field jump experiments were conducted at $2{ }^{\circ} \mathrm{C}$ to avoid field-induced denaturation of poly[d(A-T)].

The potential effect of hydrogen/deuterium replacement was tested in solutions prepared in buffer $\mathrm{A}$ in $\mathrm{H}_{2} \mathrm{O}$ initially. Then, $\mathrm{H}_{2} \mathrm{O}$ was evaporated-finally residual $\mathrm{H}_{2} \mathrm{O}$ was removed in vacuo over phosphorus pentoxide. Subsequently, the components were dissolved in the required volume of $\mathrm{D}_{2} \mathrm{O}$, and finally EtOD was added. $\mathrm{D}_{2} \mathrm{O}$ was from Sigma Aldrich, EtOD from Deutero GmbH.

Processes induced by electric field pulses were measured in a device originally constructed by Grünhagen (1974). The main parts of the high-voltage pulse generator are still as described by Grünhagen (1974), whereas other components such as the light source, measuring cell and detector were replaced. The light source used in the present experiments was a 200-W high-stability L2423 Hg/ Xe arc-lamp from Hamamatsu together with a Schoeffel GM250 grating monochromator and a Glan air polarizer. A homemade photomultiplier detector was used together with a Tektronix DSA 601A digitizer for data collection. The data were evaluated by a set of previously described programs (Diekmann et al. 1982; Porschke and Jung 1985).

Densities required for conversion between the different units used to define the composition of water-ethanol 
mixtures were taken from Haynes and Lide (2015) and from "International alcoholometric tables" (https:// www.oiml.org/en/files/pdf_r/r022-e75.pdf). Densities for $\mathrm{D}_{2} \mathrm{O}$-EtOD mixtures were measured by the DMA 602 densitometer (Anton Paar, Graz, Austria).

\section{Conditions for magic angle detection and construction of improved cell windows}

Separation of field-induced reaction effects from orientation effects requires measurements at the magic angle, which is not trivial and demands special experience. The "magic" angle refers to an orientation of polarized light at $54.8^{\circ}$ with respect to the vector of the applied electric field (Labhart 1961). Theory and experiments demonstrate that reaction effects are observed selectively at the magic angle (Porschke 1974, 1996). In practice, amplitudes should not exceed $\sim 10 \%$ of the total light intensity, because magic angle conditions may be violated otherwise. Changes in the light intensity resulting from orientation are usually much larger than those from reactions such as conformation changes; thus, magic angle conditions must be rigorously adhered to. Polarized light at the magic angle can be generated relatively easily, but the state of polarization may be seriously affected by strain in the cell windows. Such strain is induced by any mechanical stress at the optical windows. Quartz is convenient for measurements in the UV, but minor stress may cause a large birefringence, resulting in changes of the polarized light. In the past, birefringence of quartz windows was avoided by a layer of silicon grease between the cell body and the window (Porschke 1996). The viscosity of the grease had to be low enough to avoid mechanical coupling with the cell, but high enough to avoid smearing out into the cell and on the surface of the windows. In practice, cell windows had to be cleaned and reinserted frequently. These problems are avoided by a novel form of window construction and insertion into cell bodies developed recently for extension of birefringence measurements to a particularly high sensitivity (Porschke 2011). This mode of construction also proves to be very useful for absorbance measurements in field jump cells. Because arc lamps are still favorable for measurements in the UV and high light intensities are required for high time resolution, the window dimensions had to be increased. The construction used for the present measurements is shown in Fig. 1. The essential conditions for optimal implementation are: (1) selection of quartz windows completely free of strain; (2) the quartz windows are held at their outer cylinder planes by flexible O-rings made from soft silicon rubber and are not in direct contact with the cell body. Cells with optical

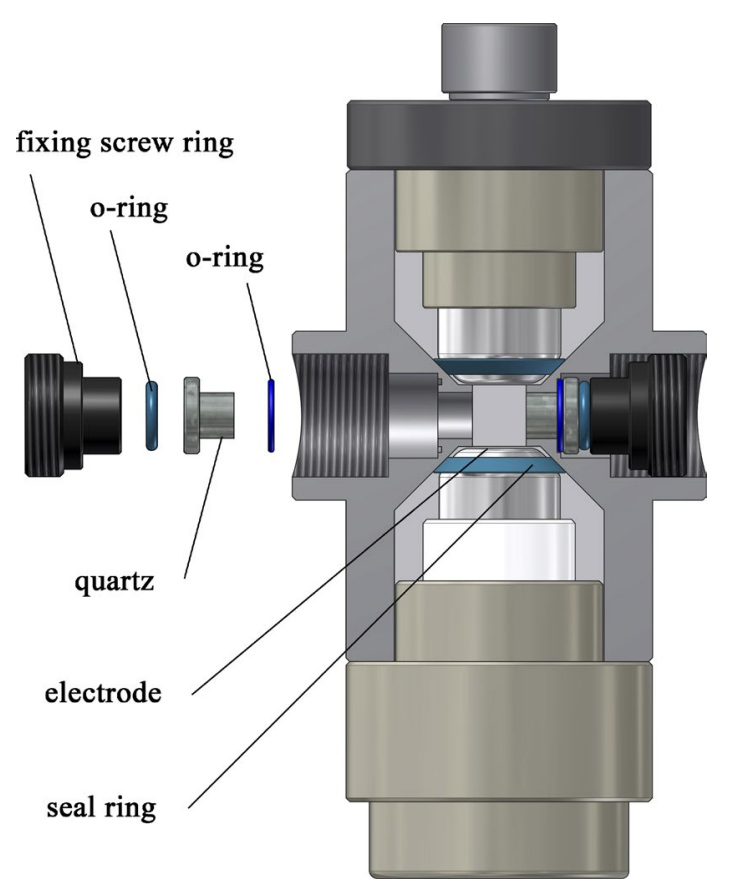

Fig. 1 Construction of the cell used for exposure of samples to electric field pulses. Form and dimensions of the cell body (macrolon) and the electrodes (platinum) are essentially as used previously. A major improvement is based on the construction of the cell windows: explosion view (left) and assembled view (right). The windows are not in direct contact with the cell body: the quartz cylinder is held at an outer segment of larger diameter between elastic O-rings (soft silicon rubber), such that the inner segment is "free" in a bore hole adjacent to the sample space

path lengths of $7 \mathrm{~mm}$ (Fig. 1) and $20 \mathrm{~mm}$ (not shown) were used in the present investigation.

\section{Results}

\section{Salt dependence}

Poly[d(AT)] samples in water-ethanol mixtures at specified buffer concentrations were subjected to DC electric field pulses, and the absorbance changes at the magic angle were recorded. As described previously (Jose and Porschke 2004, 2005), the relative absorbance changes associated with the B-A transition are particularly high at wavelengths $\geq 290 \mathrm{~nm}$. For convenient magnitudes of absolute changes in this spectral range, DNA concentrations in the range of $\sim 20$ to $\sim 280 \mu \mathrm{M}$ (monomer units) were used. Examples of field-induced transients in the range of the B-A transition are shown in Fig. 2 for the buffers A, B and C (green, blue and red line, respectively) at ethanol contents close to the centers of the B-A transition. The shape of the transients and the time constants are similar in buffers A and B. The reduction of the amplitude 


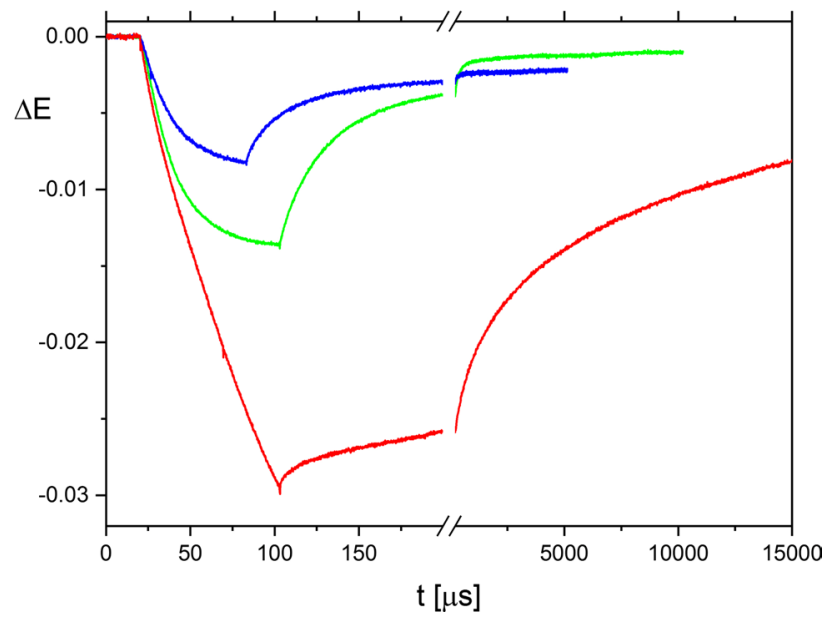

Fig. 2 Transients induced by application of electric field pulses to solutions of poly[d(AT)] at different salt concentrations: $2.44 \mathrm{mM}$ at $67.5 \mathrm{vol} \% \mathrm{EtOH}$ (buffer A, green line), $4.44 \mathrm{mM}$ at $66.71 \mathrm{vol} \% \mathrm{EtOH}$ (buffer B, blue line) and $11.4 \mathrm{mM}$ at $65.96 \mathrm{vol} \% \mathrm{EtOH}$ (buffer C, red line). The measured changes of light intensity are converted to extinction changes, which are then normalized to a concentration of $80 \mu \mathrm{M}$ phosphate units. Electric field pulses start at $t=20 \mu \mathrm{s}$; pulse lengths are 83,63 and $83 \mu$ s in buffers A, B and C, respectively; pulse amplitudes are $39 \mathrm{kV} / \mathrm{cm}$

in buffer B compared to buffer A reflects the increased electrostatic shielding resulting from the increase of the ion concentration in buffer B. A corresponding dependence was observed at the lower salt concentrations used in the previous investigation (Jose and Porschke 2004).

A detailed discussion of the processes observed upon application of the electric field and after pulse termination has been presented previously (Jose and Porschke 2004). Transients measured close to the center of the B-A transition represent large perturbations induced by the field pulses. Clear differences are observed in the reaction progress curves upon application of electric field pulses and after pulse termination. Here, discussions are restricted to reaction curves recorded after pulse termination (i.e., at electric field strength zero), representing a net reaction flow from B to A DNA. These transients show a spectrum of times with a relatively broad range of time constants. Thus, individual time constants cannot be defined as usual, and only approximate numerical values can be given. This is partly because the B-A transition is a cooperative reaction. Fitting of the transients showed a spectrum with a first process at $\sim 10 \mu$ s followed by slower components with time constants not exceeding $\sim 1 \mathrm{~ms}$.

The transient in buffer $\mathrm{B}$ indicates a shift in the $\Delta E$ level after the field jump relaxation with respect to that before application of the electric field (cf. Fig. 2). Analysis of the field jump data and comparison with measurements of the absorbance as a function of the temperature in a standard spectrophotometer demonstrate that the shifts are due to a field-induced temperature jump. This effect is minimal in buffer A because of its low conductivity, but is clearly detectable in buffer B because of its increased salt concentration.

A further increase of the salt concentration in buffer $\mathrm{C}$ leads to a clear change of the transient with respect to those found in buffers A and B: (1) the amplitude is much higher, although electrostatic shielding is increased; (2) the response time constants are much larger. These changes in the transient are accompanied by the appearance of turbidity. All these observations indicate the onset of aggregation.

The limiting value of the ethanol content, where aggregation is initiated, decreases with increasing salt concentration. At salt concentrations up to $4 \mathrm{mM}$, the B-A transition is observed before the onset of aggregation, whereas the B-A transition and aggregation appear at closely corresponding ethanol contents in $10 \mathrm{mM}$ salt.

The aggregation is not only reflected in the response to electric field pulses, but is also indicated by an increase of the absorbance due to light scattering. This effect is clearly visible, for example, at long wavelengths outside the usual absorbance band, already at $63.6 \mathrm{vol} \% \mathrm{EtOH}$ in the buffer with $10 \mathrm{mM}$ salt, whereas a minor increase was found at $63.0 \mathrm{vol} \% \mathrm{EtOH}$. At $4 \mathrm{mM}$ salt, there is no increase of the absorbance in the long wavelength range in the B-A transition range resulting from turbidity; a minor increase was observed above the B-A transition at $68.23 \mathrm{vol} \% \mathrm{EtOH}$ and a DNA concentration of $260 \mu \mathrm{M}$ (monomer units). This effect was not visible at a DNA concentration of $72.6 \mu \mathrm{M}$ (monomer units) and 68.04 vol\% EtOH content. Both EtOH content and salt concentration are particularly important parameters for aggregation. In addition, the onset of aggregation is also affected by the DNA concentration.

The field-induced change of the absorbance is maximal at the center of the B-A transition and decays to very small values outside the transition range. The dependence of the amplitudes on the ethanol content is fitted to Gaussians, providing the center and width of the transition (Fig. 3). The amplitudes were measured at different poly[d(AT)] concentrations and were normalized for fitting to a standard concentration of $80 \mu \mathrm{M}$. An extreme case is included in the data set at $4.44 \mathrm{mM}$ salt: one of the points (at 66.707 vol\% EtOH) was measured at a poly[d(AT)] concentration of $279.6 \mathrm{mM}$ and another one (at $66.775 \mathrm{vol} \% \mathrm{EtOH}$ ) at $23.1 \mu \mathrm{M}$. Although these data points were taken at widely different poly[d(AT)] concentrations, the $\Delta E$ values normalized to $80 \mu \mathrm{M}$ are very close to each other (Fig. 3). This is further evidence against aggregation effects in the BA transition range of poly[d(AT)] at $4.44 \mathrm{mM}$ salt.

There are now data for the B-A transition obtained by the electric field jump method at four different salt concentrations ranging from 0.183 to $4.44 \mathrm{mM}$ [data from the 


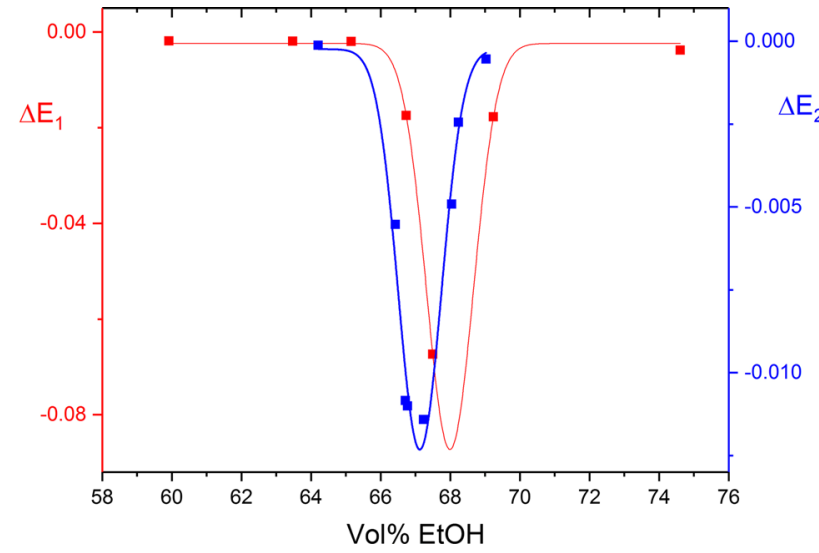

Fig. 3 Transition amplitudes induced by electric field pulses of fixed amplitude and duration in poly[d(AT)] as a function of the ethanol content at salt concentrations of $2.44 \mathrm{mM}$ (data points and $\Delta E$ scale in red, buffer A) and $4.44 \mathrm{mM}$ (data points and $\Delta E$ scale in blue, buffer B). The data are fitted to Gaussians providing the transition centers 67.99 and 67.12 together with the widths of the transition 1.59 and 1.46 for the buffers $\mathrm{A}$ and $\mathrm{B}$, respectively. $\Delta E$ values normalized to a concentration of $80 \mu \mathrm{M}$ phosphate units

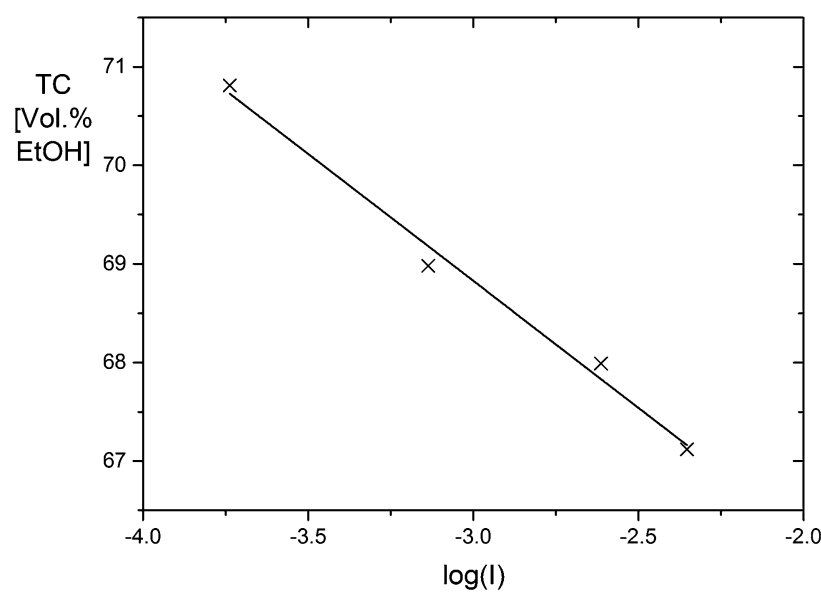

Fig. 4 Centers of the B-A transition of poly[d(AT)] as a function of the logarithm of the salt concentration. The slope obtained by linear regression is -2.57

present investigation and from Jose and Porschke (2004)]. The ethanol content at the center of the transition is a linear function of the logarithm of the salt concentration (Fig. 4). It has been suggested that the ethanol content can be used as a scale of the free energy at least approximately. Based on the assignments presented by Ivanov et al., changes in the vol\% EtOH scale may be translated to free energy changes. The slope obtained by linear regression of the data in Fig. 4 is -2.57 , corresponding to a change of vol\% EtOH by 2.57 upon a change of the salt by a factor of 10 . Using the free energy changes evaluated for three different decamer helices together with respective changes on the trifluoroethanol volume\% scale (Minchenkova et al. 1986), (vol\% TFE) provides a correspondence of $\sim 235 \mathrm{cal}$ per decamer for $1 \%$ change on the vol\% TFE scale. Each decamer helix involves nine base stacks.

Thus, a change of the salt by a factor of 10 in the case of poly[d(AT)] corresponds to $\sim 70$ cal per mole of base stack. The change of the polyelectrolyte free energy $\Delta \mathrm{G}$ for the B-A transition of double helical DNA was calculated by Manning (2002) as a function of the salt concentration $c_{i}$ : when $c_{i}$ is decreased from $10^{-2}$ to $10^{-3} \mathrm{M}, \Delta \mathrm{G}$ is increased by $\sim 100$ cal per mole base pair. Because of the approximations involved in the comparison, an exact agreement should not be expected. It may be concluded that the orders of magnitude of the experimental result and of the theoretical prediction are consistent.

\section{$\mathrm{H}_{2} \mathrm{O} / \mathrm{D}_{2} \mathrm{O}$ exchange}

The test for any dependence of the B-A transition parameters upon an exchange of the solvent from $\mathrm{H}_{2} \mathrm{O}$ to $\mathrm{D}_{2} \mathrm{O}$ was performed in buffer $\mathrm{A}$, because the field-induced amplitudes are still favorable under these salt concentration conditions. Field-induced amplitudes were measured as a function of the EtOH and EtOD content, respectively. In both cases, distinct maxima of the amplitudes were observed at the center of the B-A transition. Fitting of the data by Gaussians showed maxima at $67.7 \mathrm{vol} \% \mathrm{EtOH}$ in $\mathrm{H}_{2} \mathrm{O}$ and at $69.3 \mathrm{vol} \%$ EtOD in $\mathrm{D}_{2} \mathrm{O}$. The estimated accuracy is $\pm 0.2 \mathrm{vol} \%$. The corresponding procedure applied to the same amplitude data but using a scale based on molar concentrations of $\mathrm{H}_{2} \mathrm{O}$ and $\mathrm{D}_{2} \mathrm{O}$ provided maxima at 20.0 and $20.1 \mathrm{~mol} / \mathrm{l}$, respectively, with an estimated accuracy $\pm 0.1 \mathrm{~mol} / \mathrm{l}$. Thus, the center of the B-A transition appears at a slightly higher $\mathrm{D}_{2} \mathrm{O}$ than $\mathrm{H}_{2} \mathrm{O}$ value on the mol/l scale, but the difference is within the limit of accuracy.

Because of the sharp maximum of the amplitudes at the transition center, this center can be determined at a relatively high accuracy. This advantage does not exist for the time constants. Moreover, a special problem appears because of multiexponential transients. Separation of multiexponentials is notoriously difficult; thus, exact values of individual time constants cannot be specified. Under these conditions, the conclusion is limited to the statement that the time constants are very similar in $\mathrm{H}_{2} \mathrm{O}$ and $\mathrm{D}_{2} \mathrm{O}$.

\section{Discussion}

As demonstrated by high-resolution structures of protein DNA complexes, the B-A transition of DNA is induced upon binding of regulatory proteins to DNA in many cases (Jacobo-Molina et al. 1993; Kiefer et al. 1998; Cheetham 
and Steitz 1999; Jones et al. 1999; Lu et al. 2000). Thus, the B-A transition is directly involved in the mechanism of gene regulation. An unambiguous assignment of the steps contributing to this biologic function requires sufficient information about the B-A transition.

\section{Comparison of experimental and simulated results}

There are many publications on molecular dynamics simulations from different authors. Because of initial problems in the early phase of simulations, some conclusions about the B-A dynamics were not apparent from the beginning. Fast conversion of A-DNA to B-DNA within $\sim 1 \mathrm{~ns}$ in aqueous solutions has been simulated in many cases, whereas the opposite reaction from B- to A-DNA, expected to occur at reduced water activity, has not been observed in unbiased MD simulations (Cheatham et al. 1997; Sprous et al. 1998; Noy et al. 2007). Apparently, there is a considerable activation barrier at reduced water activity. This result may be considered consistent with the experimental data obtained at reduced water activity by the electric field jump technique (Jose and Porschke 2004, 2005) in the sense that both experiments and simulations show the existence of activation barriers at reduced water activity. However, there is no explicit information on the time scale of the B-A reaction at reduced water activity from molecular dynamics. Conversely, the field jump technique does not provide information on the B-A kinetics in usual aqueous solutions. It is remarkable that molecular dynamics simulations indicate the existence of a large activation barrier at reduced water activity and the absence of this barrier in usual aqueous environments. This contrast raises questions about the nature of the activation barrier.

The B-A transition is a special case of a stacking rearrangement, which is a frequently observed reaction in nucleic acids. A simple example is single-strand stacking, which has been studied for various cases by cable temperature jump measurements (Porschke 1976, 1977). Time constants in the range of $\sim 10 \mathrm{~ns}$ to $\sim 1 \mu$ s were observed. Corresponding results were found by laser temperature jump measurements (Dewey and Turner 1979; Freier et al. 1981). The time constant of stacking in a model compound with two adenine residues connected by a simple and flexible aliphatic $-\left(\mathrm{CH}_{2}\right)_{3}$-bridge could not be resolved by the cable temperature jump technique (Porschke 1978); thus, its stacking time constant is faster than $\sim 10 \mathrm{~ns}$. This result indicates that the "activation barrier" in the case of singlestranded stacking is imposed by the ribose- or deoxyribosephosphate backbone. Stacking is slowed down even further in more complex structures. In simple nucleic acid loops, for example, the rearrangement of stacking was found to occur with time constants $\geq 10 \mu$ s (Bujalowski et al. 1986; Menger et al. 2000). All these time constants for simple stacking rearrangements were characterized in aqueous salt solutions. Thus, the time constants observed for the B-A transition by relaxation measurements at reduced water activity are in a time range that may be expected from the time constants of usual stacking rearrangements in a usual aqueous environment. From this point of view, there is no problem regarding the nature of an activation barrier during the B-A transition.

\section{The aggregation problem}

One of the problems associated with the experimental analysis of the B-A transition has always been the decrease of DNA solubility at reduced water activity. Precipitation has been avoided simply by reduction of the salt concentration. However, the criteria for the absence of aggregation have not always been presented clearly enough. Minchenkova et al. (1986) reported that precipitation can be avoided by using trifluoroethanol (TFE) instead of ethanol for reduction of the water activity. A detailed analysis based on electro-optical measurements of rotational diffusion for natural DNA restriction fragments with mixed AT/GC sequences has been presented recently (Porschke 2016), showing a range without aggregation or condensation upon addition of ethanol at monovalent salt $\leq 1 \mathrm{mM}$. This range for analysis of the B-A transition without aggregation or condensation is extended to monovalent salt $\leq 4.4 \mathrm{mM}$ upon addition of TFE.

In the present investigation, the special effect of TFE was not used. Instead it was tested, how far the set of data available for poly[d(AT)] in $\mathrm{H}_{2} \mathrm{O}$-EtOH mixtures could be extended. The results show that an extension is possible up to a salt concentration of $4.4 \mathrm{mM}$. Strong perturbations resulting from aggregation were observed at $11.4 \mathrm{mM}$ salt concentration. The aggregation tendency of DNA without $\mathrm{GC}$ base pairs is expected to be reduced. The chain length may be another parameter affecting aggregation. Finally, the kinetics of aggregation should be considered, which is expected to be accelerated with increasing salt and ethanol content. Intermediate experimental conditions of salt and ethanol content are expected to exist where aggregation cannot be avoided at long times but remains negligible at sufficiently short times. Under these conditions there are time windows that can be used for analysis of the B-A transition.

\section{Magnitude of the electrostatic barrier}

An obvious candidate for a contribution to an activation barrier is electrostatic repulsion. The experimental analysis by measurements of the ionic strength dependence has now been extended to a wider range from 0.183 to $4.44 \mathrm{mM}$. The change of B-A transition time constants in this range is relatively small, indicating that the electrostatic contribution to the reaction barrier is not as large as may have been expected. The experimental time constants have always 
been determined close to the center of the transition. For each of the analyzed salt concentrations there is a strong dependence of the time constants on the EtOH content. The magnitude of the time constants observed under the impact of the electric field is always reduced with decreasing $\mathrm{EtOH}$ contents (Jose and Porschke 2004). However, extrapolation of these dependences to the absence of EtOH is not practicable, because the interval available for measurements is very limited; thus, extrapolation over the wide range of $\mathrm{EtOH}$ content to the absence of EtOH cannot be sufficiently accurate. Moreover, the effect of the electric field must be extrapolated as well. Under these conditions, the available experimental data can only be used with sufficient reliability for the conclusion that the time constants at the center of the transition are almost independent of the salt concentration. Because the overall activation barrier corresponds to a factor of $\sim 10^{4}$, the present results indicate that the main part of this barrier is not due to electrostatics.

\section{Solvent isotope experiment}

The $\mathrm{H}_{2} \mathrm{O} / \mathrm{D}_{2} \mathrm{O}$ exchange was conducted as a simple test for an influence of the hydrogen bonding and hydration network on the thermodynamics and kinetics of the B-A transition. The experimental data demonstrate that the B-A transition is observed at a slightly higher value on the $\mathrm{mol} / \mathrm{l}$ scale for $\mathrm{D}_{2} \mathrm{O}$ than $\mathrm{H}_{2} \mathrm{O}$, but the difference is within the limit of accuracy. Thus, the effect of the $\mathrm{H}_{2} \mathrm{O} / \mathrm{D}_{2} \mathrm{O}$ exchange on the $\mathrm{B}-\mathrm{A}$ transition is relatively small. Large effects resulting from an $\mathrm{H}_{2} \mathrm{O} / \mathrm{D}_{2} \mathrm{O}$ exchange have been reported in the literature for protein stability, dynamics and association (Cioni and Strambini 2002; Sasisanker et al. 2004; Eginton and Beckett 2013). Recent simulations on a RNA hairpin (Pathak and Bandyopadhyay 2017) indicate a large effect induced by an $\mathrm{H}_{2} \mathrm{O} / \mathrm{D}_{2} \mathrm{O}$ exchange on its thermal stability. Considering the well-documented strong influence of solvation on the B-A transition (Saenger 1984; Saenger et al. 1986), the absence of a detectable influence of the $\mathrm{H}_{2} \mathrm{O} / \mathrm{D}_{2} \mathrm{O}$ exchange is remarkable and should be analyzed in more detail.

\section{Conclusions}

An improved construction of optical windows for electric field jump cells is developed for selective characterization of field-induced reactions in the presence of orientation effects by magic angle measurements. Extension of the experimental data on the kinetics of the B-A transition in poly[d(AT)] to a wider range of salt concentrations indicates that electrostatics does not provide the main contribution to the activation barrier. The dependence of the B-A transition center on the salt concentration is in the range expected from polyelectrolyte theory. An exchange of $\mathrm{H}_{2} \mathrm{O}$ against $\mathrm{D}_{2} \mathrm{O}$ did not induce changes of the equilibrium and kinetic parameters of the B-A transition within the limits of experimental accuracy. A comparison of the experimental results for the B-A transition with those for other stacking rearrangements indicates that the B-A activation barrier found at reduced water activity is on the expected order of magnitude, whereas the absence of a barrier indicated by MD simulations for the B-A transition in the absence of ethanol appears to be unusual from this point of view.

Acknowledgements Open access funding provided by Max Planck Society. The author is indebted to Thorsten Freiberg for fine mechanical construction. The facilities of the Gesellschaft für wissenschaftliche Datenverarbeitung mbH, Göttingen, were used for data processing.

\section{Compliance with ethical standards}

Conflict of interest The author declares that he does not have a conflict of interest.

Open Access This article is distributed under the terms of the Creative Commons Attribution 4.0 International License (http://creativeco mmons.org/licenses/by/4.0/), which permits unrestricted use, distribution, and reproduction in any medium, provided you give appropriate credit to the original author(s) and the source, provide a link to the Creative Commons license, and indicate if changes were made.

\section{References}

Bujalowski W, Graeser E, Mclaughlin LW, Porschke D (1986) Anticodon loop of transfer tRNA-Phe: structure, dynamics, and $\mathrm{Mg}-2+$ binding. Biochemistry-Us 25:6365-6371

Cheatham TE, Kollman PA (1996) Observation of the A-DNA to B-DNA transition during unrestrained molecular dynamics in aqueous solution. J Mol Biol 259:434-444

Cheatham TE, Crowley MF, Fox T, Kollman PA (1997) A molecular level picture of the stabilization of A-DNA in mixed ethanol-water solutions. Proc Natl Acad Sci USA 94:9626-9630

Cheetham GMT, Steitz TA (1999) Structure of a transcribing T7 RNA polymerase initiation complex. Science 286:2305-2309

Cioni P, Strambini GB (2002) Effect of heavy water on protein flexibility. Biophys J 82:3246-3253

Dewey TG, Turner DH (1979) Laser temperature-jump study of stacking in adenylic acid polymers. Biochemistry-Us 18:5757-5762

Dickerson RE, Ng HL (2001) DNA structure from A to B. Proc Natl Acad Sci USA 98:6986-6988

Diekmann S, Hillen W, Morgeneyer B, Wells RD, Porschke D (1982) Orientation relaxation of DNA restriction fragments and the internal mobility of the double helix. Biophys Chem 15:263-270

Eginton C, Beckett D (2013) A large solvent isotope effect on protein association thermodynamics. Biochemistry-Us 52:6595-6600

Egli M, Tereshko V, Teplova M, Minasov G, Joachimiak A, Sanishvili R, Weeks CM, Miller R, Maier MA, An HY, Cook PD, Manoharan M (1998) X-ray crystallographic analysis of the hydration of A- and B-form DNA at atomic resolution. Biopolymers 48:234-252

Freier SM, Hill KO, Dewey TG, Marky LA, Breslauer KJ, Turner DH (1981) Solvent effects on the kinetics and thermodynamics of stacking in poly(cytidylic acid). Biochemistry-Us 20:1419-1426 
Grünhagen HH (1974) Entwicklung einer E-Feldsprungapparatur mit optischer Detektion und ihre Anwendung auf die Assoziation amphiphiler Elektrolyte. University of Braunschweig, Braunschweig

Haynes WM, Lide DR (2015) CRC handbook of chemistry and physics. CRC Press, Boca Raton

Ivanov VI, Minchenkova LE, Minyat EE, Frank-Kamenetski MD, Schyolkina AK (1974) The B to A transition of DNA in solution. J Mol Biol 87:817-833

Jacobo-Molina A, Ding JP, Nanni RG, Clark AD, Lu XD, Tantillo C, Williams RL, Kamer G, Ferris AL, Clark P, Hizi A, Hughes SH, Arnold E (1993) Crystal-structure of human-immunodeficiency-virus type-1 reverse-transcriptase complexed with doublestranded DNA at 3.0 angstrom resolution shows bent DNA. P Natl Acad Sci USA 90:6320-6324

Jones S, van Heyningen P, Berman HM, Thornton JM (1999) ProteinDNA interactions: a structural analysis. J Mol Biol 287:877-896

Jose D, Porschke D (2004) Dynamics of the B-A transition of DNA double helices. Nucleic Acids Res 32:2251-2258

Jose D, Porschke D (2005) The dynamics of the B-A transition of natural DNA double helices. J Am Chem Soc 127:16120-16128

Kiefer JR, Mao C, Braman JC, Beese LS (1998) Visualizing DNA replication in a catalytically active Bacillus DNA polymerase crystal. Nature 391:304-307

Knee KM, Dixit SB, Aitken CE, Ponomarev S, Beveridge DL, Mukerji I (2008) Spectroscopic and molecular dynamics evidence for a sequential mechanism for the A-to-B transition in DNA. Biophys J 95:257-272

Labhart H (1961) Methoden der Zuordnung von Absorptionsbanden von Farbstoffen zu berechneten Ubergangen. Chimia 15:20-26

Lu XJ, Shakked Z, Olson WK (2000) A-form conformational motifs in ligand-bound DNA structures. J Mol Biol 300:819-840

Manning GS (2002) Electrostatic free energy of the DNA double helix in counterion condensation theory. Biophys Chem 101:461-473

Menger M, Eckstein F, Porschke D (2000) Dynamics of the RNA hairpin GNRA tetraloop. Biochemistry-Us 39:4500-4507

Minchenkova LE, Schyolkina AK, Chernov BK, Ivanov VI (1986) CC/ GG contacts facilitate the B to A transition of DNA in solution. J Biomol Struct Dyn 4:463-476

$\mathrm{Ng}$ HL, Dickerson RE (2002) Mediation of the A/B-DNA helix transition by G-tracts in the crystal structure of duplex CATGGGCCC ATG. Nucleic Acids Res 30:4061-4067

Noy A, Perez A, Laughton CA, Orozco M (2007) Theoretical study of large conformational transitions in DNA: the $\mathrm{B} \leftrightarrow \mathrm{A}$ conformational change in water and ethanol/water. Nucleic Acids Res $35: 3330-3338$
Onsager L (1934) Deviations from Ohm's law in weak electrolytes. J Chem Phys 2:599-615

Pathak AK, Bandyopadhyay T (2017) Water isotope effect on the thermostability of a polio viral RNA hairpin: a metadynamics study. J Chem Phys 146:165104

Porschke D (1974) A conformation change of single stranded polyriboadenylate induced by an electric-field. Nucleic Acids Res 1:1601-1618

Porschke D (1976) The nature of stacking interactions in polynucleotides. molecular states in oligo- and polyribocytidylic acids by relaxation analysis. Biochemistry-Us 15:1495-1499

Porschke D (1977) Elementary steps of base recognition and helixcoil transitions in nucleic acids. Mol Biol Biochem Biophys 24:191-218

Porschke D (1978) Molecular states in single-stranded adenylate chains by relaxation analysis. Biopolymers 17:315-323

Porschke D (1996) Analysis of chemical and physical relaxation processes of polyelectrolytes by electric field pulse methods: a comparison of critical comments with facts. Ber Bunsen Phys Chem 100:715-720

Porschke D (2011) Electric birefringence at small angles from crossed position: enhanced sensitivity and special effects. J Phys Chem B 115:4177-4183

Porschke D (2016) Boundary conditions for free A-DNA in solution and the relation of local to global DNA structures at reduced water activity. Eur Biophys J Biophys Lett 45:413-421

Porschke D, Jung M (1985) The conformation of single stranded oligonucleotides and of oligonucleotide-oligopeptide complexes from their rotation relaxation in the nanosecond time range. J Biomol Struct Dyn 2:1173-1184

Saenger W (1984) Principles of nucleic acid structure. Springer, New York

Saenger W, Hunter WN, Kennard O (1986) DNA conformation is determined by economics in the hydration of phosphate groups. Nature 324:385-388

Sasisanker P, Oleinikova A, Weingartner H, Ravindra R, Winter R (2004) Solvation properties and stability of ribonuclease A in normal and deuterated water studied by dielectric relaxation and differential scanning/pressure perturbation calorimetry. Phys Chem Chem Phys 6:1899-1905

Schneider B, Patel K, Berman HM (1998) Hydration of the phosphate group in double-helical DNA. Biophys J 75:2422-2434

Sprous D, Young MA, Beveridge DL (1998) Molecular dynamics studies of the conformational preferences of a DNA double helix in water and an ethanol/water mixture: theoretical considerations of the A $\Leftrightarrow$ B transition. J Phys Chem B 102:4658-4667 